effects, and tended to have more comorbidities at MTX initiation than those who persisted on therapy.

Acknowledgement: This study was sponsored by Corrona, LLC. Corrona is supported through contracted subscriptions with multiple pharmaceutical companies. The abstract was a collaborative effort between Corrona and AbbVie with financial support provided by AbbVie.

\begin{tabular}{|c|c|c|c|c|}
\hline \multicolumn{5}{|c|}{ Clinical Disease Characteristics and Outcomes by Pattern of MTX Use } \\
\hline & Discontinued/Restarted & Discontinued & Persisted & \multirow[b]{2}{*}{ P.value } \\
\hline & $(\mathrm{N}=34)$ & $(\mathrm{N}=1 \mathrm{174})$ & $(\mathrm{N}=577)$ & \\
\hline \multicolumn{5}{|l|}{ CDAI change over 2-year follow-up } \\
\hline Change from baseline in CDAl, mean $\pm S D$ & $3.1 \pm 15.6$ & $7.4 \pm 14.9$ & $10.4 \pm 13.6$ & $<0.01$ \\
\hline Achieved LDA, $n(\%)$ & $10(29.4)$ & $58(33.3)$ & $213(36.9)$ & 0.50 \\
\hline Achieved MCID, ${ }^{\circ} n(\%)$ & $14(41.2)$ & $86(49.4)$ & $332(57.5)$ & 0.04 \\
\hline \multicolumn{5}{|c|}{ Outcomes at end of MTX use or 2-year follow-up } \\
\hline CDAl, mean $\pm S D$ & $15.6 \pm 14.4$ & $10.1 \pm 9.3$ & $8.8 \pm 9.5$ & 0.001 \\
\hline mHAQ, mean $\pm S D$ & $0.5 \pm 0.7$ & $0.4 \pm 0.5$ & $0.2 \pm 0.4$ & $<0.001$ \\
\hline Physician Global Assessment, mean \pm SD & $22.0 \pm 20.6$ & $18.1 \pm 16.4$ & $14.4 \pm 15.4$ & $<0.01$ \\
\hline Patient Global Assessment, mean $\pm S D$ & $39.4 \pm 32.5$ & $31.3 \pm 26.7$ & $25.2 \pm 25.0$ & $<0.01$ \\
\hline Pain, mean $\pm S D$ & $39.1 \pm 32.5$ & $33.9 \pm 27.3$ & $27.2 \pm 26.2$ & $<0.01$ \\
\hline Fatigue, mean $\pm S D$ & $44.0 \pm 35.0$ & $32.5 \pm 29.5$ & $27.2 \pm 26.8$ & 0.06 \\
\hline Morning stiffness, $n(\%)$ & $23(67.7)$ & $127(74.3)$ & $359(63.5)$ & 0.03 \\
\hline Morning stiffness duration (h), mean $\pm S D$ & $1.5 \pm 4.0$ & $1.3 \pm 3.2$ & $0.7 \pm 1.9$ & $<0.001$ \\
\hline \multicolumn{5}{|c|}{$\begin{array}{l}\text { CDAI, Clinical Disease Activity Index; CPP, cyclic citrullinated peptide; LDA, low disease activity; MCID, minimum } \\
\text { clinically important difference; mHAQ, modified Health Assessment Questionnaire; SD, standard deviation. }\end{array}$} \\
\hline \multicolumn{5}{|c|}{$\begin{array}{l}\text { "Achievement of LDA (CDAIS10) in those patients who initiated therapy in moderate (10<CDAIS22) or high } \\
\text { disease activity (CDAI>22). }\end{array}$} \\
\hline \multicolumn{5}{|c|}{$\begin{array}{l}{ }^{\circ} \mathrm{CDAl} \text { change }>1 \text { for those in LDA (CDAIS10) at baseline, CDAl change }>6 \text { for those in moderate disease activity } \\
(10<\mathrm{CDAl} \leq 22) \text { at baseline, and CDAI change }>12 \text { for those in high disease activity (CDAl>22) at baseline. }\end{array}$} \\
\hline
\end{tabular}

Disclosure of Interests: Leslie Harrold Shareholder of: Corrona, Grant/ research support from: Pfizer, Consultant for: AbbVie, BMS, and Genentech, Employee of: Corrona, Heather J. Litman: None declared, Jacqueline O'Brien Employee of: Corrona, Hua Feng Employee of: Corrona, Casey Schlacher Shareholder of: AbbVie, Employee of: AbbVie, Namita Tundia Shareholder of: AbbVie, Employee of: AbbVie

DOI: 10.1136/annrheumdis-2019-eular.1009

\section{FRI0153 OPHTHALMOLOGICAL ADVERSE EVENTS UNDER JAK INHIBITORS IN PATIENTS WITH RHEUMATOID ARTHRITIS: CASE ANALYSIS OF THE EUROPEAN PHARMACOVIGILANCE DATABASE}

Sophie Hecquet ${ }^{1}$, Marie Blanche Rabier ${ }^{1}$, Marion Lepelley ${ }^{2}$, Frank Verhoeven ${ }^{1}$, Anne Sophie Gauthier ${ }^{1}$, Clément Prati ${ }^{1}$, Bernard Delbosc ${ }^{1}$, Daniel Wendling ${ }^{1}$.

${ }^{1}$ Centre hospitalier régional universitaire de Besançon, Besançon, France; ${ }^{2}$ Centre Hospitalier Universitaire de Grenoble, La Tronche, France

Background: Ophthalmological manifestations in rheumatoid arthritis (RA) include dry syndrome, scleritis, episcleritis, uveitis and peripheral ulcerative keratitis (PUK). Recently, a new class of synthetic molecules has been developed, called JAK inhibitors (JAKinhib). We observed one case of PUK occurring two months after the introduction of baricitinib treatment with corneal perforation with secondary improvement after discontinuation of JAKinhib.

Objectives: The aim of this study is to describe and characterize the ophthalmological side effects of JAK inhibitors (JAK inhib) in patients with rheumatoid arthritis (RA) from European pharmacovigilance (PV) data.

Methods: The ophthalmological manifestations that appeared under JAK inhib were extracted from the European PV database (May 2018), EUDRAVIGILANCE following a request addressed to the National Drug Safety Agency carried out according to the following MedDRA classification "SOC eye disorders and BARICITINIB" and "SOC eye disorders and TOFACITINIB".

Results: A total of 41 patients with ophthalmic adverse events (AEs) on JAK inhib were reported. Among patients, 12 were treated with BARICITINIB and 29 with TOFACITINIB. Of these AEs, $51 \%$ were reported by the medical profession, $39 \%$ by patients and $10 \%$ by paramedical teams. The database analysis revealed 4 scleritis, 3 uveitis, 1 PUK and 1 corneal ulceration. The average time to onset of AEs was 13.2 months. The infectious causes had been eliminated.

\begin{tabular}{|c|c|c|c|}
\hline \multicolumn{4}{|l|}{ Ocular adverse events under JAK inhibitors } \\
\hline & $\begin{array}{c}\text { BARICITINIB } \\
n=12\end{array}$ & $\begin{array}{c}\text { TOFACITINIB } \\
n=29\end{array}$ & $\begin{array}{c}\text { Mean } \\
\text { Delay } \\
\text { (in days) }\end{array}$ \\
\hline Ophthalmological events & AEs & AEs & \\
\hline Scleritis $^{\star \star}, \mathrm{n}(\%)$ & $2(17)$ & $2(7)$ & 344 \\
\hline Corneal ulceration ${ }^{\star \star}, \mathrm{n}(\%)$ & 0 & $1(3)$ & 90 \\
\hline $\mathrm{PUK}^{\star \star}, \mathrm{n}(\%)$ & $1(8)$ & 0 & 57 \\
\hline Uveitis $^{\star \star}, \mathrm{n}(\%)$ & 0 & $3(10)$ & 838 \\
\hline Blurry vision*, $\mathrm{n}(\%)$ & $3(25)$ & $8(28)$ & 9 \\
\hline Red eyes*, n (\%) & $3(25)$ & $1(3)$ & 33 \\
\hline Dry eyes* ${ }^{*}, \mathrm{n}(\%)$ & $2(17)$ & $3(10)$ & NA \\
\hline Photophobia* $^{*}$ n (\%) & 0 & $1(3)$ & NA \\
\hline Decreased visual acuity ${ }^{*}, \mathrm{n}(\%)$ & $1(8)$ & $1(3)$ & 124 \\
\hline Retinal detachment ${ }^{\star \star}, \mathrm{n}(\%)$ & 0 & $1(3)$ & 480 \\
\hline Retinal haemorrhage ${ }^{\star \star}, \mathrm{n}(\%)$ & 0 & $2(7)$ & 541 \\
\hline Cataract $^{\star \star}, n(\%)$ & $2(17)$ & $5(17)$ & 413 \\
\hline Glaucoma**, n (\%) & 0 & $4(14)$ & 489 \\
\hline Age related macular degeneration ${ }^{* *}, \mathrm{n}$ & 0 & $1(3)$ & NA \\
\hline
\end{tabular}

(\%)

${ }^{*}$ Adverse event reported by the patient ${ }^{* *}$ Adverse event reported by the medical profession PUK: Peripheral Ulcerative Keratitis NA: Not Available

Conclusion: Ophthalmological manifestations under JAKinhib seem to be rare but not exceptional, the rheumatologist must be aware of them in order to discuss the imputability of the treatment. A discontinuation of it seems justified pending an ophthalmological opinion.

Disclosure of Interests: None declared

DOI: 10.1136/annrheumdis-2019-eular.3811

\section{FRI0154 SAFETY AND EFFICACY OF FILGOTINIB IN PATIENTS AGED 65 YEARS AND OLDER: RESULTS FROM A PHASE 3 STUDY IN PATIENTS WITH ACTIVE RHEUMATOID ARTHRITIS AND PRIOR INADEQUATE RESPONSE OR INTOLERANCE TO BIOLOGICAL DMARDS (BDMARD-IR)}

Kenneth Kalunian ${ }^{1}$, Jacques-Eric Gottenberg ${ }^{2}$, Mark C. Genovese ${ }^{3}$, Neelufar Mozaffarian ${ }^{4}$, Beatrix Bartok ${ }^{4}$, Franziska Matzkies ${ }^{4}$, Jie Gao ${ }^{4}$, Ying Guo ${ }^{4}$, Tsutomu Takeuchi ${ }^{5}$, Kurt de Vlam ${ }^{6}$, David Walker ${ }^{7}{ }^{1}$ University of California, San Diego, La Jolla, United States of America; ${ }^{2}$ Dept of Rheumatology, Strasbourg University Hospital, Strasbourg, France; ${ }^{3}$ Stanford University Medical Center, Palo Alto, United States of America; ${ }^{4}$ Gilead Sciences, Inc., Foster City, United States of America; ${ }^{5}$ Keio University School of Medicine, Tokyo, Japan; ${ }^{6}$ Universitair Ziekenhuis Leuven, Leuven, Belgium; ${ }^{7}$ Northumbria Healthcare, Newcastle upon Tyne, United Kingdom

Background: Filgotinib (FIL), an oral selective Janus kinase 1 (JAK1) inhibitor, demonstrated efficacy and safety vs placebo (PBO) in bDMARDIR patients with active RA in a global phase 3 study (FINCH2, ClinicalTrials.gov NCT02873936).

Objectives: We performed a prespecified subgroup analysis of the safety and efficacy of FIL in patients aged $\geq 65$ years vs $<65$ years in FINCH2 to understand FIL effects in older patients.

Methods: 449 patients were randomized 1:1:1 to FIL $200 \mathrm{mg}$, FIL 100 $\mathrm{mg}$, or PBO on a background of CsDMARDs for 24 weeks. The primary efficacy endpoint was ACR20 response at week 12 .

Results: Of 448 patients who received $\geq 1$ dose of study drugs, 113 $(25.2 \%)$ were $\geq 65$ years of age; baseline disease characteristics were similar for both age groups. Safety and efficacy parameters by age group are in Tables 1 and 2. The most common treatment-emergent adverse events (TEAEs, by System Organ Class) category was infections and infestations; nasopharyngitis, upper respiratory tract infections, and bronchitis were the most common infections and infestations in the FIL arms in both age groups. There were no cases of opportunistic infection, active tuberculosis, malignancy, gastrointestinal perforation, or death. Efficacy outcomes were similar for both groups.

Conclusion: Overall, older age was not associated with higher incidence of safety events (serious infections, herpes zoster, MACE occurred in the group $<65 \mathrm{yrs}$ ) and efficacy was similar in older and younger bDMARDIR patients with active RA.

REFERENCE:

[1] MC Genovese, et al. ACR, 2018. Abstract L06. 
Table 1. TEAEs and Key Safety Outcomes Week 0-24 by Age Group, $\mathrm{n}(\%)$

\begin{tabular}{lcccccc}
\hline & \multicolumn{3}{c}{$<65$ yrs $(\mathrm{n}=335)$} & \multicolumn{3}{c}{$\geq 65$ yrs $(\mathrm{n}=113)$} \\
\hline Regimen & $\begin{array}{c}\text { FIL 200 } \\
(\mathrm{n}=112)\end{array}$ & $\begin{array}{c}\text { FIL 100 } \\
(\mathrm{n}=117)\end{array}$ & $\begin{array}{c}\text { PBO } \\
(\mathrm{n}=106)\end{array}$ & $\begin{array}{c}\text { FIL 200 } \\
(\mathrm{n}=35)\end{array}$ & $\begin{array}{c}\text { FIL 100 } \\
(\mathrm{n}=36)\end{array}$ & $\begin{array}{c}\text { PBO } \\
(\mathrm{n}=42)\end{array}$ \\
\hline TEAE & 72 & 81 & 70 & 30 & 16 & 30 \\
& $(64.3)$ & $(69.2 \%)$ & $(66.0 \%)$ & $(85.7 \%)$ & $(44.4 \%)$ & $(71.4 \%)$ \\
Serious AE & $4(3.6)$ & $7(6.0 \%)$ & $4(3.8 \%)$ & $2(5.7 \%)$ & $1(2.8 \%)$ & $1(2.4 \%)$ \\
TEAE leading to study drug & $4(3.6)$ & $6(5.1 \%)$ & $1(0.9 \%)$ & $1(2.9 \%)$ & 0 & $2(4.8 \%)$ \\
discontinuation & & & & & & \\
Infection & 39 & 47 & 27 & 14 & 5 & 11 \\
& $(34.8)$ & $(40.2 \%)$ & $(25.5 \%)$ & $(40.0 \%)$ & $(13.9 \%)$ & $(26.2 \%)$ \\
Herpes Zoster & $2(1.8)$ & $2(1.7)$ & 0 & 0 & 0 & 0 \\
(uncomplicated) & & & & & & \\
Serious infection & $1(0.9)$ & $3(2.6)$ & $2(1.9)$ & 0 & 0 & 0 \\
MACE & 0 & $1(0.9)$ & $1(0.9)$ & 0 & 0 & 0 \\
Retinal vein occlusion & $1(0.9)$ & 0 & 0 & 0 & 0 & 0 \\
\hline
\end{tabular}

Table 2. Week 24 Key Efficacy Measures by Age Group

\begin{tabular}{lcccccc}
\hline & \multicolumn{3}{c}{$<65$ yrs $(\mathrm{n}=335)$} & \multicolumn{3}{c}{$\geq 65$ yrs $(\mathrm{n}=113)$} \\
\hline Regimen & $\begin{array}{l}\text { FIL 200 } \\
(\mathrm{n}=112)\end{array}$ & $\begin{array}{c}\text { FIL 100 } \\
(\mathrm{n}=117)\end{array}$ & $\begin{array}{c}\text { PBO } \\
(\mathrm{n}=106)\end{array}$ & $\begin{array}{c}\text { FIL 200 } \\
(\mathrm{n}=35)\end{array}$ & $\begin{array}{c}\text { FIL 100 } \\
(\mathrm{n}=36)\end{array}$ & $\begin{array}{c}\text { PBO } \\
(\mathrm{n}=42)\end{array}$ \\
\hline ACR20 $\mathrm{n}(\%)^{1}$ & $78(70) \dagger$ & $60(51) \dagger$ & $33(31)$ & $24(69)^{*}$ & $24(67)^{*}$ & $18(43)$ \\
DAS28(CRP)<2.6 $\mathrm{n}$ & $33(30) \ddagger$ & $26(22)^{*}$ & $11(10)$ & $12(34)$ & $14(39)^{*}$ & $7(17)$ \\
$(\%)^{1}$ & & & & & & \\
HAQ-DI mean CFB & -0.75 & -0.60 & -0.40 & -0.74 & -0.59 & -0.48 \\
$(\mathrm{SD})$ & $(0.66) \dagger$ & $(0.66)^{*}$ & $(0.58)$ & $(0.48)^{*}$ & $(0.67)$ & $(0.65)$ \\
SF-36 PCS mean CFB & $9.4(8.6) \dagger$ & $9.2(8.7) \dagger$ & $6.2(8.2)$ & $9.4(6.7)^{*}$ & $8.5(7.8)$ & $7.5(7.4)$ \\
(SD) & & & & & & \\
FACIT-Fatigue mean & 11.2 & 9.6 & 7.2 & 12.9 & 10.3 & 6.4 \\
CFB (SD) & $(11.6) \dagger$ & $(11.1)$ & $(10.1)$ & $(12.2)^{*}$ & $(8.3)$ & $(10.7)$ \\
\hline
\end{tabular}

${ }^{*} \mathrm{p}<0.05,{ }^{\dagger} \mathrm{p}<0.01, \$ \mathrm{p}<0.001 \mathrm{CFB}$, change from BL. ${ }^{1}$ Nonresponder imputation

Disclosure of Interests: Kenneth Kalunian Grant/research support from: Gilead Sciences, Inc., Jacques-Eric Gottenberg Grant/research support from: Bristol-Myers Squibb, Grant/research support from: Bristol-Myers Squibb, Consultant for: Bristol-Myers Squibb, Lilly, Pfizer, Sanofi-Genzyme, UCB Pharma, Consultant for: Bristol-Myers Squibb, Eli Lilly, UCB, SanofiGenzyme, Pfizer, Mark C. Genovese Grant/research support from: Sanofi/ Genzyme, Genentech/Roche, RPharm, Consultant for: Sanofi/Genzyme, Genentech/Roche, RPharm, Neelufar Mozaffarian Shareholder of: Gilead, Employee of: Gilead, Beatrix Bartok Shareholder of: Gilead, Employee of: Gilead, Franziska Matzkies Shareholder of: Gilead, Employee of: Gilead, Jie Gao Shareholder of: Gilead, Employee of: Gilead, Ying Guo Shareholder of: Gilead, Employee of: Gilead, Tsutomu Takeuchi Grant/research support from: Astellas Pharma Inc, Chugai Pharmaceutical Co, Ltd., Daiichi Sankyo Co., Ltd., Takeda Pharmaceutical Co., Ltd., AbbVie GK, Asahikasei Pharma Corp., Mitsubishi Tanabe Pharma Co., Pfizer Japan Inc., Eisai Co., Ltd., AYUMI Pharmaceutical Corporation, Nipponkayaku Co. Ltd., Novartis Pharma K.K., Grant/research support from: AbbVie, Asahi Kasei, Astellas, AstraZeneca, AYUMI, Bristol-Myers Squibb, Chugai, Daiichi Sankyo, Eisai, Eli Lilly Japan, Janssen, Mitsubishi Tanabe, Nippon Kayaku, Novartis, Pfizer Japan Inc, Taiho, Taisho Toyama, Takeda, Teijin, Grant/research support from: Astellas Pharma Inc., Bristol Myers Squibb, Chugai Pharmaceutical Co., Ltd., Mitsubishi Tanabe Pharma Co., Pfizer Japan Inc., Santen Pharmaceutical Co., Ltd., Takeda Pharmaceutical Co., Ltd., Teijin Pharma Ltd., AbbVie GK, Asahi Kasei Pharma Corp., Taisho Toyama Pharmaceutical Co., Ltd., SymBio Pharmaceuticals Ltd., Janssen Pharmaceutical K.K., Celltrion Inc., Nipponkayaku Co. Ltd., and UCB Japan, Consultant for: Astra Zeneca K.K., Eli Lilly Japan K.K., Novartis Pharma K.K., Mitsubishi Tanabe Pharma Co., Abbivie GK, Nipponkayaku Co.Ltd, Janssen Pharmaceutical K.K., Astellas Pharma Inc., Taiho Pharmaceutical Co. Ltd., Chugai Pharmaceutical Co. Ltd., Taisho Toyama Pharmaceutical Co. Ltd., GlaxoSmithKline K.K., UCB Japan Co. Ltd., Consultant for: AbbVie, Asahi Kasei, Astellas, AstraZeneca, AYUMI, Bristol-Myers Squibb, Chugai, Daiichi Sankyo, Eisai, Eli Lilly Japan, Janssen, Mitsubishi Tanabe, Nippon Kayaku, Novartis, Pfizer Japan Inc, Taiho, Taisho Toyama, Takeda, Teijin, Consultant for: Astra Zeneca K.K., Eli Lilly Japan K.K., Novartis Pharma K.K., Mitsubishi Tanabe Pharma Co., Asahi Kasei Medical K.K., AbbVie GK, Daiichi Sankyo Co., Ltd., Bristol Myers Squibb, and Nipponkayaku Co. Ltd., Speakers bureau: Astellas Pharma Inc., Bristol Myers Squibb, Chugai Pharmaceutical Co., Ltd., Mitsubishi Tanabe Pharma Co., Pfizer Japan Inc., Santen Pharmaceutical Co., Ltd., Takeda Pharmaceutical Co., Ltd., Teijin Pharma Ltd., AbbVie GK, Asahi Kasei Pharma Corp., Taisho Toyama Pharmaceutical Co., Ltd., SymBio Pharmaceuticals Ltd., Janssen Pharmaceutical K.K., Celltrion Inc., Nipponkayaku Co. Ltd., and UCB Japan, Speakers bureau: AbbVie, Asahi Kasei, Astellas, AstraZeneca, AYUMI, Bristol-Myers Squibb, Chugai,
Daiichi Sankyo, Eisai, Eli Lilly Japan, Janssen, Mitsubishi Tanabe, Nippon Kayaku, Novartis, Pfizer Japan Inc, Taiho, Taisho Toyama, Takeda, Teijin, Speakers bureau: AbbVie GK., Bristol-Myers K.K., Chugai Pharmaceutical Co. Ltd., Mitsubishi Tanabe Pharma Co., Pfizer Japan Inc., Astellas Pharma Inc, Diaichi Sankyo Co. Ltd., Eisai Co. Ltd., Sanofi K.K., Teijin Pharma Ltd., Takeda Pharmaceutical Co. Ltd., Novartis Pharma K.K., Kurt de Vlam Consultant for: Pfizer Inc, Consultant for: Johnson \& Johnson, David Walker: None declared

DOI: 10.1136/annrheumdis-2019-eular.2121

\section{FRI0155 A COMPARISON OF UPADACITINIB PLUS METHOTREXATE AND UPADACITINIB PLUS OTHER CSDMARDS IN PATIENTS WITH RHEUMATOID ARTHRITIS: AN ANALYSIS OF TWO PHASE 3 STUDIES}

Joel Kremer ${ }^{1}$, Filip van den Bosch ${ }^{2}$, Andrea Rubbert-Roth ${ }^{3}$, Sebastião C. Radominski ${ }^{4}$, Gerd Rüdiger Burmester ${ }^{5}$, Heidi Camp ${ }^{6}$, Sebastian Meerwein ${ }^{7}$, Mark Howard ${ }^{6}$, Yanna Song ${ }^{6}$, Sheng Zhong ${ }^{6}$, Bernard Combe ${ }^{8} .{ }^{1}$ Albany Medical College, Albany, United States of America; ${ }^{2}$ Ghent University Hospital and Ghent University, Ghent, Belgium; ${ }^{3}$ Kantonsspital St Gallen, St Gallen, Switzerland; ${ }^{4}$ Universidade Federal do Paraná, Curitiba, Brazil; ${ }^{5}$ Charité-Universitätsmedizin, Berlin, Germany; ${ }^{6}$ AbbVie Inc., North Chicago, United States of America; ${ }^{7}$ AbbVie Deutschland, Ludwigshafen, Germany; ${ }^{8} \mathrm{CHU}$ Montpellier, Université de Montpellier, Montpellier, France

Background: Upadacitinib (UPA), a selective JAK1 inhibitor, has shown efficacy in patients with rheumatoid arthritis (RA) when combined with methotrexate (MTX) or other conventional synthetic disease-modifying antirheumatic drugs (csDMARDs). ${ }^{1,2}$ However, the efficacy of UPA plus MTX has not been directly compared with UPA plus other csDMARDs.

Objectives: To compare the efficacy of UPA in combination with MTX versus UPA in combination with other csDMARDs in patients with an inadequate response (IR) to csDMARDs (SELECT-NEXT ${ }^{1}$ ) or biologic DMARDs (bDMARDs; SELECT-BEYOND ${ }^{2}$ ).

Methods: 661 patients in SELECT-NEXT and 498 patients in SELECTBEYOND received UPA $15 \mathrm{mg}$ or $30 \mathrm{mg}$ once daily (QD) or placebo (PBO) for 12 weeks; all patients received concomitant $\operatorname{csDMARD(s).~The~}$ primary endpoints for both studies were rates of ACR20 response and DAS28(CRP) $\leq 3.2$. Additional endpoints included DAS28(CRP) $<2.6$, CDAl low disease activity $(\leq 10)$, and CDAI remission $(\leq 2.8)$. Patients were grouped according to concomitant csDMARD use (MTX vs non-MTX csDMARDs); patients receiving both MTX and a non-MTX csDMARD were included in the MTX group. p-values were calculated based on a logistic regression model with treatment group and type of background csDMARD as fixed factors, adjusting for demographic and baseline factors. p-values from the interaction between treatment group and background csDMARD were also calculated, to assess the consistency of the effects of study treatments for different background csDMARD type. Nonresponder imputation was used for missing data.

Results: In SELECT-NEXT and SELECT-BEYOND, 535 and 410 patients, respectively (80\%), were receiving concomitant MTX (mean dose $17 \mathrm{mg} /$ week), and 124 and 82 patients were receiving non-MTX csDMARDs. Demographics and disease characteristics were broadly similar between treatment groups; the majority of patients were female and of white ethnicity, and around half were using oral corticosteroids at baseline. Across all subgroups, the proportion of patients achieving efficacy outcomes was higher with both UPA doses compared with PBO (Table). There were no significant differences between efficacy outcomes with UPA in combination with MTX versus UPA in combination with non-MTX csDMARDs in either patient population. This included ACR20 response as well as low disease activity and remission defined by DAS28(CRP) and CDAl.

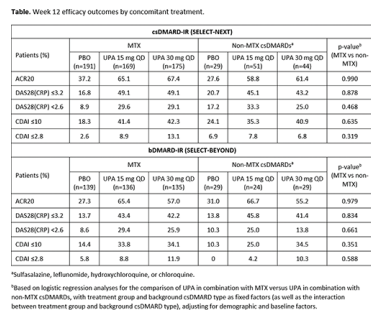

Conclusion: In this post hoc analysis, the efficacy of UPA in patients with RA appeared comparable whether administered in combination with MTX or non-MTX csDMARDs. 